\title{
Gestion intégrée de l'environnement en milieu urbain : vers un renouvellement des pratiques planificatrices?
}

\author{
Mario Gauthier ${ }^{1}$ \\ Institut d'urbanisme, Université de Montréal
}

\section{Introduction}

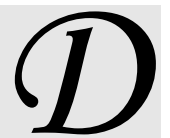
ans le domaine de la planification urbaine, de l'aménagement du territoire et de la gestion de l'environnement, la question de l'intégration s'impose de plus en plus comme un impératif. La notion d'intégration est au cœur du concept de développement durable qui renvoie justement à l'idée de concilier les dimensions écologiques, sociales et économiques du développement, de même qu'à articuler les échelles territoriales (du local au global) et temporelles (du court au long terme). Cependant, malgré l'engouement récent pour la question de l'intégration en matière de développement durable, la thématique de la «gestion intégrée » n'est pas nouvelle; elle s'inscrit dans le courant de remise en cause du modèle de la planification rationnelle en cours depuis les années d'après-guerre et a donné lieu à de multiples mises en pratique.

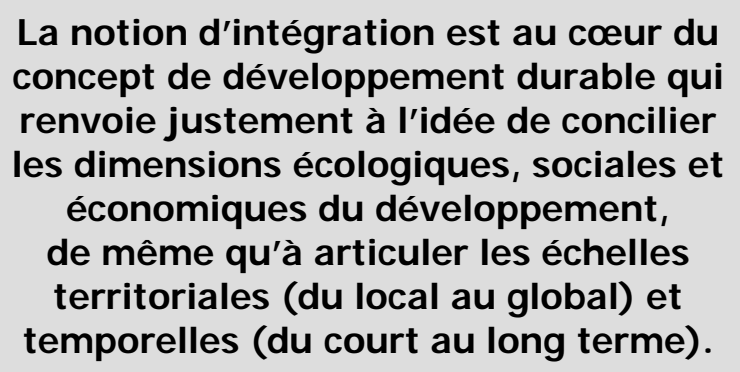

Afin de contribuer à la réflexion sur la notion d'intégration dans le champ des études urbaines et environnementales, ce texte présente d'abord l'origine et les assises théoriques du concept de gestion intégrée de l'environnement, propose des définitions et décrit ses principales dimensions. La deuxième partie du texte examine trois expériences pionnières afin de situer le contexte d'émergence du modèle québécois de gestion intégrée : le Bureau d'aménagement de l'est du Québec (BAEQ) et l'aménagement intégrée des ressources dans le Bas du Fleuve, le Plan d'aménagement de la rivière Yamaska à proximité de Montréal et le projet Archipel dans la région de Montréal. La troisième partie du texte passe en re$\mathrm{Au}$ Québec, dans la foulée de plusieurs expériences internationales, dont celle de la Tennessee Valley Authority ${ }^{2}$, diverses initiatives de " gestion intégrée » - des ressources, de l'environnement et des territoires - ont été mises de l'avant avec plus ou moins de succès.

Toutefois, si la question de l'intégration n'est pas nouvelle, la généralisation du concept et des pratiques se réclamant de la "gestion intégrée » soulève plusieurs interrogations: qu'est-ce que la gestion intégrée des ressources, de l'environnement et des territoires? Quelle est la portée théorique et pratique de cette notion sur le plan des pratiques planificatrices ? Quelles sont les leçons à tirer des expériences qué- vue trois exemples d'expérimentations récentes développées en marge de la procédure québécoise d'évaluation environnementale : une médiation portant sur un projet de construction au chantier maritime de Lévis, près de Québec, une concertation au moment de l'élaboration d'un projet de restauration environnementale dans le Port de Montréal et une consultation publique sur l'amélioration de la mobilité dans la région de Montréal.

Ces trois expériences ont en commun de viser à résoudre des problèmes complexes d'environnement en décloisonnant les approches trop sectorielles et en favorisant la négociation entre les acteurs. La conclusion propose une discussion sur la portée théorique et 
pratique de ces expériences de gestion intégrée pour contribuer au renouvellement des pratiques planificatrices.

\section{La gestion centralisée, sectorielle, fragmentée et centrée sur des objectifs préétablis par les planificateurs conduit à des solutions peu adaptées aux particularités locales.}

\section{La gestion intégrée de l'environnement : origine, assises théoriques, définitions et principales dimensions}

\section{Origine et assisses théoriques}

La thématique de la gestion intégrée de l'environnement s'est imposée progressivement dans les années d'après-guerre en réaction au modèle rationnel de prise de décision (rational comprehensive planning) qui suppose un lien direct entre la connaissance scientifique d'un problème environnemental et l'action publique pour y répondre ${ }^{3}$. Les principales caractéristiques de la gestion conventionnelle des ressources et de l'environnement, particulièrement la quête de l'optimisation et de la meilleure solution possible (one best way), sont alors critiquées et mises en cause non seulement par les spécialistes des sciences sociales, mais également par les professionnels eux-mêmes. La rationalité guidant la recherche de la solution optimale s'avère généralement étroite et incapable de prendre en compte la complexité des problématiques environnementales. Le caractère global et exhaustif du processus de planification, qui passe par l'analyse en profondeur de toutes les options et l'évaluation de leurs conséquences, entraîne une lourdeur bureaucratique (coûts, durée, etc.) peu propice à la résolution des problèmes. Le processus de prise de décision incrémental, par essais-erreurs-corrections, conduit à des ajustements à la marge nettement insuffisants pour résoudre les problèmes complexes. Les solutions proposées sont généralement fondées sur l'expertise des professionnels (ingénieurs, planificateurs, etc.) sans nécessairement tenir compte des valeurs et des intérêts divergents des usagers du territoire. La gestion centralisée, sectorielle, fragmentée et centrée sur des objectifs préétablis par les planificateurs conduit à des solutions peu adaptées aux particularités locales. De plus, le modèle rationnel amène les planificateurs à surestimer la capacité prévisionnelle de la planification et à sous-estimer les difficultés de mise en œuvre sur le terrain. On observe alors des écarts considérables entre les intentions initiales et la gestion effective de l'environnement.

En réaction aux échecs et aux insuffisances du modèle rationnel de prise de décision, le courant communicationnel au sein des théories de la planification propose une démarche de planification interactive axée sur la concertation et la négociation entre les acteurs (stakeholders) ${ }^{4}$. Selon ce courant, le dialogue entre les acteurs est essentiel pour définir des valeurs et des intérêts communs nécessaires à la résolution des problématiques environnementales. Pour ce faire, les tenants de la planification interactive préconisent un recours à des stratégies visant à favoriser les échanges entre les parties prenantes; la concertation, la négociation, la médiation, la recherche de consensus et la délibération apparaissent ainsi comme des dispositifs essentiels pour organiser les discussions entre les acteurs. Selon ce courant de planification, l'interaction entre les acteurs représente le meilleur moyen pour arrimer les connaissances scientifiques des problèmes d'environnement à l'action publique pour les résoudre. La planification devient un processus interactif et politique. Pour ce faire, le courant de la planification interactive propose de revoir la relation entre les planificateurs et les usagers du territoire en conférant au planificateur un rôle de médiateur qui consiste à favoriser des débats ouverts, informés et argumentés dans la but d'atteindre une solution acceptable et appuyée par l'ensemble des parties prenantes. De ce point de vue, la planification renvoie à un processus favorisant la négociation autour de l'intérêt général. Son succès est mesuré par l'atteinte d'un accord, d'une entente ou d'une solution négociée à l'échelle du territoire. Cette conception procédurale de la planification est au cœur du concept de gestion intégrée de l'environnement.

\section{Définitions et principales dimensions}

À l'instar du concept de développement durable, la notion de «gestion intégrée de l'environnement » est polysémique ${ }^{5}$. Toutefois, on reconnaît généralement que cette notion s'est développée dans la foulée des initiatives visant à intégrer la planification, l'évaluation et la gestion des ressources (Integrated Resource Planning) et qu'elle désigne une approche stratégique et interactive qui vise à faire en sorte qu'une mul- 
titude de besoins et de valeurs soient pris en compte dans le processus décisionnel. Ainsi, la gestion intégrée - des ressources, de l'environnement et des territoires - renvoie essentiellement à des procédures et à des démarches de discussion, de débat public et de concertation entre les acteurs, dont l'objectif est d'établir une compréhension commune des problèmes à traiter et de développer des plans intégrés et partagés de gestion à l'échelle des territoires. Dans la pratique, une grande diversité d'acteurs se réunissent régulièrement pour partager informations et points de vue, établir une compréhension mutuelle des problèmes et éventuellement s'entendre sur une action concertée pour les résoudre.

Les diverses définitions de la gestion intégrée de l'environnement comprennent généralement quatre dimensions: holistique, systémique, stratégique et orientée vers l'action (figure 1).

Les deux premières dimensions (holistique et systémique) renvoient au contenu, aux objectifs et aux ambitions de l'intégration. L'approche est holistique parce qu'elle adopte une vision large de la question en prenant en considération les différents aspects du problème à résoudre (écologique, économique, social). Elle est également systémique en ce sens qu'elle cherche à prendre en compte les interrelations entre les composantes biophysiques et humaines du système environnemental.

Ces deux dimensions de la gestion intégrée invitent les acteurs à adopter une compréhension écosystémique et une approche interdisciplinaire pour la résolution des problèmes environnementaux.

\section{Figure 1 - Contenu et procédure de la gestion intégrée de l'environnement}

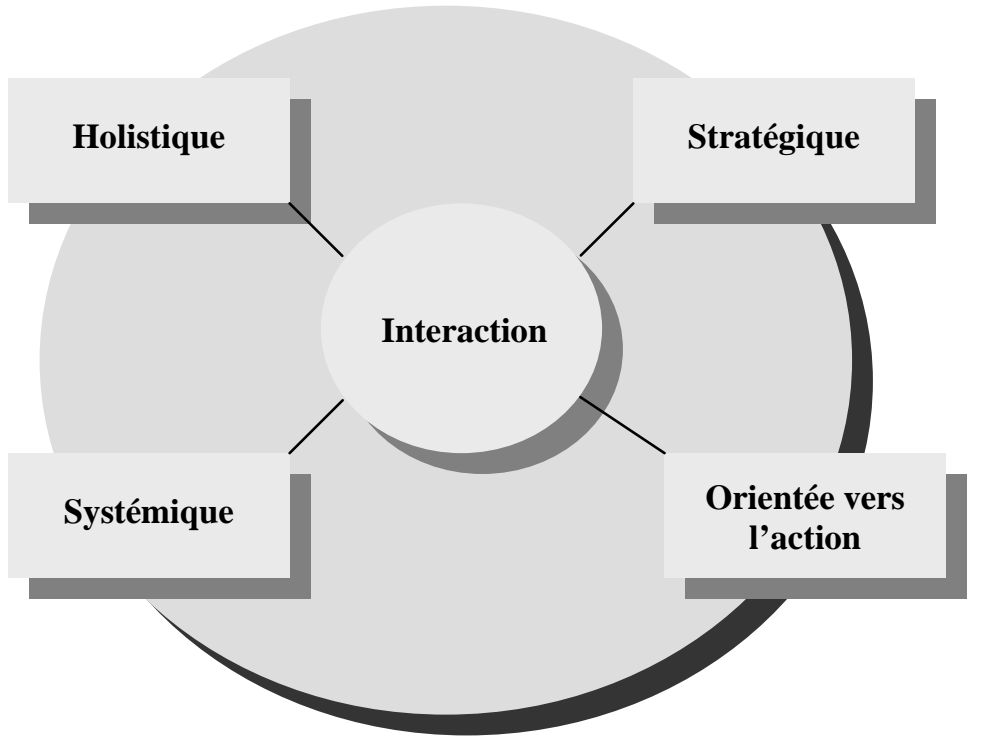

D'après Hooper et Margerum (2000).

Les dimensions stratégique et orientée vers l'action de la gestion intégrée renvoient aux procédures, aux instruments et aux moyens de mise en œuvre. Elles invitent les acteurs à concentrer leurs actions et leurs énergies sur les enjeux prioritaires et à adopter une démarche proactive orientée vers la mise en œuvre. Elles visent à réduire l'écart entre la planification et la mise en œuvre (implementation gap) qui caractérise la gestion conventionnelle de l'environnement.

Pour faciliter ce travail de repérage des éléments prioritaires à considérer, diverses méthodes de cadrage basées sur la participation des acteurs en amont du processus décisionnel sont proposées. La gestion intégrée est considérée stratégique et orientée vers l'action lorsque les divers acteurs adoptent une démarche proactive axée sur l'état désiré de l'environnement en fixant des objectifs réalistes qui tiennent compte des moyens de mise en œuvre.

Cette démarche pragmatique, qui tient compte des contraintes et des opportunités de l'action, se veut suffisamment flexible pour composer avec le changement et l'incertitude. Enfin, l'interaction entre les 
acteurs, à travers des procédures de discussion, de débat public et de concertation, apparaît comme le meilleur moyen pour articuler ces quatre dimensions.

\section{L'émergence d'un modèle québécois de gestion intégrée : les expériences pionnières}

Afin de rendre compte du contexte d'émergence d'un modèle québécois de gestion intégrée et de la traduction de ce concept dans la pratique, cette section examine trois expériences pionnières en matière de gestion intégrée de l'environnement: le Bureau d'aménagement de l'est du Québec (BAEQ) et les projets d'aménagement intégré des ressources dans le Bas du Fleuve, le Plan d'aménagement de la rivière Yamaska à proximité de Montréal et le projet Archipel dans la région de Montréal. La description et l'analyse rétrospective de ces expériences permettent de dégager quelques enseignements sur le plan du contenu (objectifs et ambitions) et des procédures (instruments et moyens d'actions).

\section{Le BAEQ et l'aménagement intégré des ressources}

L'expérience du Bureau d'aménagement de l'est du Québec (BAEQ) au début des années 1960 représente l'un des premiers exercices de planification régionale et d'aménagement du territoire au Québec ${ }^{6}$. Chargé d'élaborer un plan intégré de développement, le BAEQ s'est appuyé sur une enquête/participation conduite par des jeunes professionnels des sciences sociales. Dans son Plan de développement de l'est du Québec, déposé en juin 1966, le BAEQ dresse un constat d'échec devant le déclin agricole et forestier de l'arrière-pays et propose, dans une optique de modernisation économique, la fermeture de certaines parties du territoire en déplaçant les populations dans des centres urbains plus dynamiques.

Cette expérience de planification, décrite comme « une entreprise d'éducation à la rationalité » et d' « animation sociale» a donné lieu, au début des années 1970, à des luttes et à des contestations importantes qui ont suscité l'émergence de projets communautaires d'aménagement intégré des ressources. Ces projets de remise en valeur des terres agricoles et forestières ont été développés en réaction au plan d'aménagement proposé par le BAEQ visant à rationaliser l'exploitation des ressources. Refusant la fermeture de leurs territoires, les organismes commu-

nautaires ont mis en place une Société régionale d'aménagement intégré des ressources possédant des fonctions de coordination et de concertation entre les acteurs. Les projets mis de l'avant se sont appuyés sur l'idée d'une exploitation rationnelle et intégrée de toutes les ressources du territoire en fonction d'un contrôle local. Même si ces expériences de relance des activités agricoles et forestières ne se sont pas avérées concluantes, près de trente ans plus tard les recherches sur l'évolution agricole de l'arrière-pays bas-laurentien font état de la consolidation d'une agroforesterie en marge d'une agriculture moderne.

Le Plan Yamaska s'est inscrit en continuité avec l'approche fonctionnelle et instrumentale du territoire.

\section{Le Plan d'aménagement de la Rivière Yamaska}

Le Plan d'aménagement de la Rivière Yamaska, réalisé sous la coordination de l'Office de planification et de développement du Québec (OPDQ) au début des années 1970, représente une première expérimentation de gestion intégrée de l'eau par bassin versant au Québec. Pour élaborer le Plan Yamaska, l'OPDQ a initié la création d'une instance de coordination politique (un comité d'orientation interministériel) s'appuyant sur une équipe technique de mise en œuvre (comité d'exécution). La mise en place d'une instance de participation municipale sous la forme d'un comité consultatif a également été envisagée sans toutefois être concrétisée. De sorte que le Plan Yamaska, déposé en 1972, est essentiellement un projet d'experts gouvernementaux qui s'est résumé à la mise en place d'équipements visant la protection contre les inondations, la régularisation des eaux, le drainage des terres agricoles, la protection des rives contre l'érosion et l'assainissement des eaux. Ce faisant, le Plan Yamaska s'est inscrit en continuité avec l'approche fonctionnelle et instrumentale du territoire qui a caractérisé les travaux de régularisation des eaux réalisés par les entreprises privées de production hydroélectrique au début du $\mathrm{XX}^{\mathrm{e}}$ siècle.

L'étude rétrospective de cette expérience pionnière réalisée par Jean-François Bibeault ${ }^{7}$ met en lumière les difficultés liées à la mise en œuvre de la gestion intégrée de l'eau par bassin versant au Québec. Son analyse de cette expérimentation montre clairement le 
passage d'une conception techniquement intégrée au moment de la planification à une fragmentation au moment de la mise en œuvre, révélée par la désarticulation et l'abandon de certains projets. Elle dévoile aussi les contradictions entre une logique d'efficacité globale des interventions portée par le ministère des Ressources naturelles et les diverses logiques sectorielles des autres ministères. De sorte que, « dans ce jeu d'acteurs institutionnels, on arrive parfois à des compromis, parfois à des décisions imposées sans concertation ni coordination. La puissance d'un acteur institutionnel couplée à son réseau social lui permet ainsi de s'imposer au détriment d'acteurs moins biens organisés, moins soutenus par une collectivité et financièrement moins bien pourvus ${ }^{8}$.

\section{Le projet Archipel dans la région de Montréal}

Le projet Archipel, tel que conçu par le gouvernement du Québec au début des années 1980, se présente comme un vaste programme d'aménagement des divers plans d'eau de la région de Montréal ${ }^{9}$. Initialement créé pour répondre aux problèmes d'inondations, le projet est devenu un projet d'aménagement hydraulique à fins multiples. Le projet initial, né de la rencontre entre des objectifs de protection contre les inondations et des volontés de développer le potentiel hydroélectrique du plan d'eau montréalais, prévoyait la construction par Hydro-Québec d'une centrale hydroélectrique à la hauteur des rapides de Lachine et un contrôle des débits et des niveaux d'eau dans une perspective de conciliation des usages. Les objectifs mis de l'avant par les instances officielles sont multiples et potentiellement conflictuels: contrôle des inondations, amélioration de la qualité de l'eau, amélioration de l'accessibilité aux rives et aux plans d'eau, protection des écosystèmes, mise en valeur du potentiel hydroélectrique, navigation commerciale, préservation et mise en valeur du patrimoine.

\section{Le projet Archipel a avorté, en juin 1982, au moment de la tenue de la Table de concertation.}

Afin d'harmoniser ces objectifs et de concilier les usages, les concepteurs du projet Archipel envisageaient un processus de concertation continue entre les divers usagers de la ressource. Jean Décarie et Gilles Boileau ${ }^{10}$ résument bien l'esprit et le processus de conciliation des intérêts recherchés : «Les responsables sociaux de chacun des éléments constitutifs de l'écosystème sont appelés, concrètement, à venir défendre et développer leurs intérêts particuliers et à s'entendre sur l'intérêt général, sur un rééquilibrage $\mathrm{du}$ système comme tel, assuré par une gestion collective de la ressource commune $»$. Selon eux, l'étude de faisabilité du projet Archipel se présente comme une étude d'impact inversée puisque "à partir d'une hypothèse de projet collectif, on demande aux partenaires sociaux de réagir, de formuler leurs intentions comme leurs inquiétudes, qui devront être satisfaites, conciliées, intégrées jusqu'à ce qu'un projet acceptable, et donc faisable, émerge et se dessine plus précisément ». Toutefois, le projet Archipel a avorté, en juin 1982, au moment de la tenue de la Table de concertation, en raison justement de l'incapacité à réunir les conditions de la concertation, et à maîtriser en un même temps les conditions du marché de l'énergie. À ce moment, les élus municipaux et les principaux groupes socio-économiques ont exprimé des réticences à l'aménagement d'une centrale hydroélectrique dans les rapides de Lachine, et Hydro-Québec s'est retiré du projet en invoquant des raisons de rentabilité économique. Le projet Archipel a toutefois eu pour principale retombée la mise en place d'un projet de réseau d'espaces verts et bleus dans la région métropolitaine de Montréal.

\section{Leçons des expériences pionnières}

Que nous apprennent ces expériences pionnières en matière de planification et de gestion intégrée du territoire ? L'expérience du BAEQ nous enseigne d'abord que la participation envisagée comme un exercice d'éducation populaire et de persuasion sociale joue un rôle négligeable sur le contenu de la planification. En effet, la démarche d'enquête/participation du BAEQ s'est avérée une démarche de consultation à sens unique qui visait à changer les mentalités de la population et des usagers du territoire, dont les agriculteurs et les travailleurs de la forêt. Le processus de participation des usagers s'est transformé en une simple expérience d'éducation populaire, de sorte que le processus n'a pas permis de concilier les intérêts divergents des principaux acteurs (citoyens, élus locaux, usagers, planificateurs, gestionnaires, etc.) au moment de l'élaboration du plan. Toutefois, l'expérience de participation du BAEQ a donné lieu à une importante mobilisation de la population qui semble avoir favo- 
risé, dans les décennies suivantes, la consolidation d'une agroforesterie dans le Bas-du-Fleuve.

En ce qui concerne les deux expériences pionnières de gestion intégrée de l'eau - le Plan Yamaska et le projet Archipel -, elles nous révèlent la tension constante entre, d'une part, les ambitions démesurées de l'intégration au moment de la planification et, d'autre part, la fragmentation des actions lors de la mise en œuvre. Ces expérimentations témoignent également des difficultés liées à la création d'un espace de coordination interministériel et de concertation des acteurs locaux pour prendre en compte la complexité et assurer la conciliation des intérêts divergents. De plus, elles montrent que la prégnance d'un acteur à l'étape de la planification - le ministère des Ressources naturelles dans le cas du Plan Yamaska et Hydro-Québec dans le cas du projet Archipel - entraîne souvent des problèmes liés à la hiérarchisation des objectifs ainsi que des résistances au moment de la mise en œuvre. En effet, la perspective d'implantation d'équipements et de mise en œuvre des projets - le caractère stratégique et orienté vers l'action - s'oppose souvent à la perspective de concertation visant à concilier des objectifs divergents. Enfin, ces deux expériences de gestion intégrée de l'eau sous-tendent une conception de l'intérêt général négocié localement, sans pour autant avoir prévu de procédures pour faciliter la négociation, l'harmonisation des intérêts divergents et la résolution des conflits.

\section{Trois expériences récentes en marge de la procédure québécoise d'évaluation environnementale}

Contrairement aux expériences pionnières examinées précédemment, les grands projets d'équipements assujettis à la procédure québécoise d'évaluation environnementale sont confrontés à des exigences formeles de planification et de gestion, à savoir la réalisation d'une étude des impacts sur l'environnement et la tenue d'audiences publiques sous l'égide du Bureau d'audiences publiques sur l'environnement (BAPE). En outre, en raison de l'importance accordée à la participation des publics, l'approche québécoise d'évaluation environnementale est souvent considérée comme un modèle en matière de gestion de l'environnement et d'aménagement du territoire. Toutefois, malgré ses forces reconnues - rigueur, clarté, accessibilité, transparence, crédibilité, etc. - cette procédure

présente aussi de nombreuses faiblesses : les audiences publiques interviennent tardivement dans le processus décisionnel, elles confinent le public dans une position réactive et on leur reproche d'être longues, coûteuses et de favoriser les conflits et les oppositions. Les démarches de médiation, de concertation au moment de l'élaboration des projets et de consultation sur les politiques se sont développées pour palier ces insuffisances. À travers l'examen de trois cas récents, l'objectif de cette section est de présenter ces innovations procédurales visant à assurer une gestion intégrée de l'environnement.

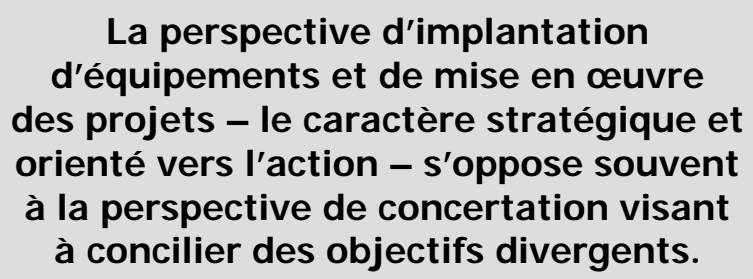

\section{La médiation environnementale : le cas d'un projet de construction d'une cour d'entreposage d'acier au chantier maritime MIL Davie, à Lévis}

Afin d'assurer sa compétitivité sur le marché international et de stabiliser son niveau d'emploi, la compagnie MIL Davie a entrepris, au milieu des années 1990, de réorienter ses activités en construisant des navires marchands (pétroliers et vraquiers). Pour ce faire, la compagnie a élaboré un projet qui consiste essentiellement à améliorer la productivité de son chantier maritime situé à Lévis, près de Québec, en aménageant une cour d'entreposage d'acier et deux nouveaux ateliers. Le projet initial, qui impliquait un remblayage important dans le fleuve Saint-Laurent, aurait entraîné la destruction d'une partie d'un herbier (marais de l'Anse-aux-Sauvages), la perte d'un habitat reconnu pour la faune et la destruction d'un boisé en bordure du marais. La réalisation du projet aurait également eu des effets importants sur le paysage en limitant l'accès visuel aux secteurs de Beauport et de la Côte-de-Beaupré.

La médiation environnementale ${ }^{11}$ entreprise par le BAPE à la suite des demandes d'audiences publiques de trois organismes préoccupés par la conservation des milieux humides a permis aux parties de ratifier une entente. Celle-ci prévoit, entre autres, que le promoteur s'engage à acheter du Port de Québec un 
marais de 27 hectares (l'Anse Gilmour) et à le céder à un organisme voué à la conservation de ce territoire. La compagnie s'est également engagée à ne réaliser que la première phase de son projet sur sa propriété de l'Anse-aux-Sauvages, à soumettre la seconde phase à l'analyse d'experts indépendants et à appuyer la tenue d'une consultation publique sur tout nouveau projet dans cette zone. En contrepartie, les groupes écologistes acceptent de reconnaître le bien-fondé de la réalisation du projet et renoncent à leur droit à une audience publique sur le remblai fluvial. En somme, la démarche de médiation a permis aux parties prenantes de s'entendre non seulement sur le bien-fondé de la réalisation du projet, mais également sur la protection et la mise en valeur des milieux naturels situés à proximité du chantier maritime de Lévis.

\section{La concertation à l'étape de l'élaboration du projet : le cas de la restauration environnementale d'un secteur de la zone portuaire de Montréal}

Au début des années 1990, à la suite des épisodes de remise en suspension des sédiments contaminés dans un secteur du Port de Montréal et dans le cadre de son Plan d'action Saint-Laurent, Environnement Canada a suscité la formation d'un groupe de travail dans le but de réaliser une restauration environnementale du site ${ }^{12}$. Ce groupe de travail, composé de représentants de l'administration portuaire de Montréal et des entreprises riveraines (une usine d'affinage des métaux et des pétrolières), a réalisé plusieurs études et s'est transformé par la suite en Groupe de restauration afin de développer un projet commun de décontamination $\mathrm{du}$ site. Le Groupe de restauration s'est ensuite adjoint un Comité consultatif chargé de favoriser le consensus entre les entreprises concernées et de susciter la participation de la population au processus décisionnel. Le Comité consultatif, initié par la Comité ZIP (Zone d'intervention prioritaire) JacquesCartier, réunit des représentants socio-économiques (comités de citoyens, associations de défense de l'environnement, etc.), des représentants des gouvernements (fédéral, provincial et municipal) ainsi que les membres du Groupe de restauration.

Les échanges et les négociations entre ces divers intervenants, qui se sont échelonnés sur plusieurs années, ont essentiellement porté sur les engagements financiers des entreprises et du Port de Montréal, sur la nature et le niveau de décontamination à atteindre, les options de traitement et la gestion des sédiments suite à leur retrait du lit du fleuve. Les travaux conjoints du Groupe de restauration et du Comité consultatif ont également favorisé l'expression des préoccupations de la population en ce qui a trait au mode de traitement des sédiments (exportation des sédiments traités, confinement des sédiments, etc.), à la gestion des nuisances pendant les travaux (odeurs, transport des sédiments, etc.) ainsi qu'aux modalités d'information, de consultation publique et de suivi environnemental du projet. Les résultats de cette démarche de concertation au moment de l'élaboration du projet sont de plusieurs ordres. La concertation a d'abord favorisé la formation du Groupe de restauration réunissant les entreprises industrielles et le Port de Montréal. Les négociations entre les acteurs ont également permis la ratification d'une entente liant le Groupe de restauration et les gouvernements sur un projet de restauration environnementale évalué à 6,9 M\$ qui serait complété à l'été 2006. Le projet a également fait l'objet d'une évaluation des impacts sur l'environnement en concertation avec les acteurs en amont des audiences publiques formelles du BAPE. En outre, le projet prévoit une démarche active de suivi environnemental associant les principaux acteurs concernés.

\section{La consultation sur une politique : les travaux de la Commission de consultation sur l'amélioration de la mobilité entre Montréal et la Rive-Sud}

Dans le contexte des grandes orientations et des objectifs énoncés dans le Plan de gestion des déplacements de la région métropolitaine de Montréal, adopté en 2000, le ministère des Transports du Québec a créé, en janvier 2001, une commission chargée d'examiner l'opportunité d'établir un nouveau lien de transport reliant l'île de Montréal à la Rive-Sud ${ }^{13}$. Cette solution avait été identifiée pour répondre au problème de la congestion routière et améliorer la fluidité de la circulation vers le centre-ville de l'agglomération. Toutefois, dès le départ, les activités de consultation se sont situées en amont des projets concrets d'infrastructures routières à l'étape stratégique de la planification, de sorte que les débats se sont rapidement élargis à l'examen d'enjeux stratégiques tels que la gestion de la demande, le financement des infrastructures, l'émission des gaz à effet de serre, la qualité de vie et la santé.

Les différentes activités d'évaluation et de consultation qui se sont échelonnées sur une période de deux 
ans - rencontres thématiques, sondages, études d'experts, audiences publiques, etc. - ont permis aux acteurs de mieux cerner la nature et l'ampleur des problèmes reliés à la mobilité entre Montréal et la Rive-Sud. De la sorte, les travaux de la commission ont rempli une fonction de cadrage des enjeux en identifiant les préoccupations et les problèmes importants et en précisant l'information nécessaire à la prise de décision ainsi que les incertitudes. Ils ont également favorisé l'établissement d'un consensus sur la nécessité d'élaborer un cadre de travail intégré combinant un éventail de solutions incluant les transports en commun, les investissements routiers et la gestion de la demande. Enfin, ils ont permis d'identifier les projets routiers et de transports en commun susceptibles d'être réalisés. En somme, les travaux de la commission ont favorisé les discussions sur les grandes orientations stratégiques, les options, les combinaisons de solutions et l'éventail des scénarios et ce, dans un contexte d'interaction entre les acteurs et de participation publique situé en amont du processus décisionnel et à l'échelle du territoire métropolitain.

\section{Conclusion}

L'appel croissant en faveur d'une gestion intégrée des ressources, de l'environnement et des territoires et la multiplication des pratiques s'en réclamant soulèvent plusieurs interrogations sur le plan des pratiques planificatrices. Si le concept est relativement bien défini et possède de bonnes assises théoriques, il n'en demeure pas moins que, dans la pratique, la gestion intégrée n'est pas facile à mettre en œuvre. Alors que les expériences pionnières se sont avérées plus ou moins fructueuses en raison des déficiences sur le plan procédural, il est permis de se demander si les expériences récentes ne misent justement pas trop sur des procédures d'interactions entre les acteurs médiation, concertation, consultation - au détriment du contenu, des objectifs et des ambitions de l'intégration. En effet, pour les planificateurs et les gestionnaires urbains, l'intégration signifie non seulement de répondre aux enjeux et aux défis de la coordination interministérielle et de l'interaction entre les acteurs, mais aussi aux impératifs d'une vision holistique et écosystémique des problématiques d'aménagement. En ce sens, la gestion intégrée ne peut se résumer à prévoir des procédures de concertation, de débat public ou de médiation; elle doit également s'assurer de développer des outils et des moyens de planification (études, diagnostics, scénarios, évaluations, etc.) aptes à favoriser l'intégration. En définitive, le défi de la de la gestion intégrée de l'environnement en milieu urbain est double : penser à la fois le contenu et les procédures de l'intégration.

\section{La gestion intégrée ne peut se résumer à prévoir des procédures de concertation, de débat public ou de médiation.}

\section{Remerciements}

L'auteur remercie Gérard Beaudet de l'Institut d'urbanisme de l'Université de Montréal et Jean-François Bibeault du Centre Saint-Laurent de Environnement Canada pour leurs commentaires et suggestions sur une première version de ce texte.

\section{Notes et références}

1 Mario Gauthier est professeur invité à l'Institut d'urbanisme de la Faculté de l'aménagement de l'Université de Montréal.

2 Dans le contexte de la Grande Dépression des années 1930, le président des États-Unis, Franklin Roosevelt, a demandé au Congrès américain de créer une agence innovatrice, la Tennessee Valley Authority, chargée d'assurer une gestion intégrée des ressources de ce bassin versant en adoptant une démarche de résolution de problèmes et de conciliation des usages (production hydroélectrique, navigation, contrôle des inondations, érosion des sols, prévention de la malaria, etc.). Pour plus de details, voir : A Short History of TVA. From the New Deal to a New Century (http://www.tva.gov/abouttva/history.htm).

3 Sur la crise du modèle de la planification rationnelle et la critique de la raison technique et scientifique, voir: Alexander, E.R. (1984). "After Rationality, What? A Review of Responses to Paradigm Breakdown », Journal of American Planning Association, vol. 50, $\mathrm{n}^{\circ}$ 1, p. 62-69; Hamel, P. (1996). « Crise de la rationalité : le modèle de la planification rationnelle et les rapports entre connaissance et action », dans R. Tessier et J.-G. Vaillancourt (dir.), La recherche sociale en environnement: nouveaux paradigmes, Montréal, Presses de l'Université de Montréal, p. 6174.

4 La planification interactive renvoie au tournant communicationnel au sein des théories de la planification qui s'inspire de la philosophie de l'agir communicationnel de Jürgen Habermas. Pour plus de détails, voir: Hamel, P. (1997). « La critique post-moderne et le courant communi- 
cationnel au sein des théories de la planification: une rencontre difficile », Les Cahiers de géographie du Québec, vol 41, n 114, p. 311-322 ; Healey, P. (1993). «Planning Through Debate: The Communicative Turn in Planning Theory », dans F. Fischer et J. Forester (dir.), The Argumentative Turn in Policy Analysis and Planning, Durham and London, Duke University Press, p. 233-253.

5 Sur le concept de gestion intégrée de l'environnement, voir entre autres : Born, S.M. et W.C. Sonzogni (1995). «Integrated Environmental Management: Strengthening the Conceptualization », Environmental Management, vol. 19, $\mathrm{n}^{\circ}$ 2, p. 167-181; Hooper, B.P. et R.D. Margerum (2000). «Integrated Watershed Management for River Conservation : Perspectives from Experiences in Australia and the United States », dans P.J. Boon, B.R. Davies et G.E. Petts (dir.), Global Perspective on River Conservation : Science, Policy and Practice, John Wiley \& Sons, p. 509-517; Margerum, R.D. (1997). «Integrated Approaches to Environmental Planning and Management », Journal of Planning Literature, vol. 11, $\mathrm{n}^{\circ}$ 4, p. 459-475; Margerum, R.D. (1999). «Integrated Environmental Management : the Foundation for Successful Practice », Environmental Management, vol. 24, $\mathrm{n}^{\circ}$ 2, p. 151-166; Margerum, R.D. et S.M. Born (1995). « Integrated Environmental Management: Moving from Theory to Practice », Journal of Environmental Planning and Management, vol. $38, \mathrm{n}^{\circ} 3$, p. 371-390.

6 Sur le BAEQ et les projets d'aménagement intégrée des ressources dans le Bas-du-Fleuve, voir: Anger, P. et L. Dion (1971). Participation et planification régionale: l'expérience du Bureau d'aménagement de l'est du Québec (1963-1966), rapport de recherche, Université Laval, 319 p; Dionne, H. et al. (1983). Aménagement intégré des ressources et luttes en milieu rural, Université du Québec à Rimouski, Groupe de recherche interdisciplinaire en développement de l'Est du Québec, 351 p; Godbout, J,T. (1983). La participation contre la démocratie, Montréal, Éditions Saint-Martin, 190 p; Jean, B. (1997). Territoires d'avenir. Pour une sociologie de la ruralité, Sainte-Foy, PUQ, $318 \mathrm{p}$.

7 Bibeault, J.-F. (1997). « L'émergence d'un modèle québécois de gestion de l'eau à la rencontre des territoires et des réseaux », dans M. Gariépy et M. Marié (dir.), Ces réseaux qui nous gouvernent? Montréal, L'Harmattan, p. 325-343; Bibeault, J.-F. (2003). «La gestion intégrée de l'eau : dynamique d'acteurs, de territoires et de techniques », Les Cahiers de géographie du Québec, vol. 47, n 132, p. 389411.
8 Bibeault, J.-F. (2003), op. cit., p. 403.

9 Sur le projet Archipel dans la région de Montréal, voir : Décarie, J. (1987). « L'archipel après Archipel », conférence "Hydro-Québec » de la Faculté de l'aménagement de l'Université de Montréal, 3 novembre; Décarie, J. et G. Boileau (1983). «Le projet Archipel : une réflexion et une discussion géographiques », Les Cahiers de géographie du Québec, vol. 27, n 71, p. 323-340; Soubeyran, O. (1986). «L'évolution de la planification environnementale en matière de loisirs touristiques à Montréal : vers un aménagement intégrée de "l'espace bleu ?" ", dans P. Georges (dir.), La géographie du Canada, Presses universitaires de Bordeaux, p. 199-214.

10 Décarie, J. et G. Boileau (1983), op. cit., p. 330 et 331.

11 Pour une analyse exhaustive de cette innovation procédurale et un bilan de l'expérience québécoise de médiation environnementale, voir: Gauthier, M. (1998). Participation du public à l'évaluation environnementale : une analyse comparative d'études de cas de médiation environnementale, thèse de doctorat en études urbaines, UQAM, 317 p.; sur le projet de la compagnie MIL Davie, le lecteur pourra se référer au rapport d'enquête et de médiation $\mathrm{n}^{\circ}$ 96 du Bureau d'audiences publiques sur l'environnement.

12 Pour une analyse détaillée de cette expérience de concertation, voir: Lepage, L., M. Gauthier et P. Champagne, (2003). «Le projet de restauration du fleuve SaintLaurent: de l'approche technocratique à l'implication des communautés riveraines $»$, Sociologies Pratiques, $\mathrm{n}^{\circ} 7, \mathrm{p}$. 65-89; sur le projet de restauration environnementale du secteur 103 de la zone portuaire de Montréal, le lecteur pourra consulter l'étude d'impact sur l'environnement déposée au ministre de l'Environnement du Québec (Desseau Soprin, mai 2004) ainsi que le compte rendu de la période d'information et de consultation publiques du BAPE tenue du 18 mai au 2 juillet 2004.

13 Pour plus de détails sur les travaux de la commission, voir : Gauthier, M., L. Lepage et S. Tremblay (2002). Stratégies d'intervention pour améliorer la mobilité entre Montréal et la Rive-Sud: évaluation environnementale stratégique et concertation en amont du processus décisionnel, rapport final déposé à la Commission de consultation sur l'amélioration de la mobilité entre Montréal et la Rive-Sud, Chaire d'études sur les écosystèmes urbains, UQAM, septembre, 45 p.; Québec (2003). Mieux se déplacer entre Montréal et la Rive-Sud, Commission de consultation sur l'amélioration de la mobilité entre Montréal et la Rive-Sud, Gouvernement du Québec, 197 p. 


\section{Publicité}

« Réseau VRM » 